\title{
Linx
}

Revue des linguistes de l'université Paris X Nanterre

$45 \mid 2001$

Invariants et variables dans les langues. Études typologiques

\section{Remarques sur la notion de passif et l'origine des constructions passives}

Denis Creissels

\section{(2) OpenEdition}

\section{Journals}

Édition électronique

URL : http://journals.openedition.org/linx/772

DOI : $10.4000 /$ linx.772

ISSN : 2118-9692

Éditeur

Presses universitaires de Paris Nanterre

Édition imprimée

Date de publication : 1 décembre 2001

Pagination : 71-82

ISSN : 0246-8743

Référence électronique

Denis Creissels, "Remarques sur la notion de passif et l'origine des constructions passives », Linx [En ligne], 45 | 2001, mis en ligne le 19 juin 2012, consulté le 10 décembre 2020. URL : http:// journals.openedition.org/linx/772; DOI : https://doi.org/10.4000/linx.772

Ce document a été généré automatiquement le 10 décembre 2020.

Département de Sciences du langage, Université Paris Ouest 


\title{
Remarques sur la notion de passif et l'origine des constructions passives
}

\author{
Denis Creissels
}

1 L'approche typologique n'a de sens que dans la mesure où on s'efforce d'appliquer de façon cohérente les termes grammaticaux à toutes les langues prises en compte dans la comparaison. Il est bien sûr souhaitable de s'écarter le moins possible de leur usage traditionnel. Mais, une fois dégagée une définition générale qui doit s'efforcer d'être compatible avec l'usage d'un maximum de linguistes décrivant les langues, l'entreprise typologique n'a de sens que si l'on est prêt à reconnaître, d'une part des cas où l'usage traditionnel du terme en question s'écarte de la définition retenue pour la comparaison des langues, d'autre part des cas où le terme en question n'est pas traditionnellement utilisé, mais où la définition retenue pour la comparaison des langues justifierait son introduction.

2 Ce problème se pose notamment avec acuité pour les notions de passif et de moyen, qui sont au centre de cet exposé. Avant d'entrer dans le vif du sujet, il sera donc nécessaire de procéder à une tentative de mettre un peu d'ordre dans les pratiques terminologiques.

\section{La notion de passif : passif canonique et passifs non canoniques, voix oblique et voix impersonnelle}

3 Des travaux sur le passif se dégage clairement une notion de passif canonique défini comme modification morphologique du verbe liée à la « destitution » de l'argument traité comme sujet ${ }^{1}$ dans la construction de base et à la "promotion » de l'argument traité comme objet ${ }^{2}$ dans la construction de base du verbe, traditionnellement désignée comme active. Ceci veut dire qu'au passif, l'argument qui serait traité comme sujet des formes dites actives peut, soit disparaitre totalement de la construction du verbe, soit se maintenir avec le statut d'oblique, tandis que l'argument qui serait traité comme objet des formes dites actives prend au passif le statut de sujet. Les phrases (b) des ex. (1) à (3) illustrent cette notion. 


\begin{tabular}{|c|c|c|c|c|c|}
\hline (1) & \multicolumn{5}{|c|}{ tswana ${ }^{3}$} \\
\hline & a. & \multicolumn{3}{|l|}{ Ke-tlaa-kwala } & Lokwalo \\
\hline & & \multicolumn{3}{|l|}{ IS1SG-FUT- écrire } & 11lettre \\
\hline & & \multicolumn{3}{|l|}{ 'J'écrirai une lettre' } & \\
\hline & b. & \multicolumn{3}{|l|}{ Lokwalo } & lo-tlaa-kwal-wa \\
\hline & & \multicolumn{3}{|l|}{ 11lettre } & ISCL11-FUT-écrire-PSF \\
\hline & & \multicolumn{4}{|l|}{ 'La lettre sera écrite' } \\
\hline (2) & \multicolumn{5}{|c|}{ peul } \\
\hline \multirow{2}{*}{\multicolumn{2}{|c|}{ a }} & Aali & hocc-ii & sawru & ndu \\
\hline & & Ali & ramasser-ACC & bâton & le \\
\hline & & \multicolumn{4}{|l|}{ Ali a ramassé le bâton' } \\
\hline & b. & Sawru & ndu & \multicolumn{2}{|c|}{ hocc-aama } \\
\hline & & bâton & le & \multicolumn{2}{|c|}{ ramasser-ACC.PSF } \\
\hline & & \multicolumn{4}{|c|}{ 'Le bâton a été ramassé' } \\
\hline (3) & \multicolumn{5}{|c|}{ k'ichee' } \\
\hline & a. & X-e-ki-kunaaj & ri & alab'oom & ri \\
\hline & & ACC-IO3PL-IS3PL-soigner & les & enfants & les \\
\hline & \multicolumn{5}{|c|}{ 'Les femmes ont soigné les enfants' } \\
\hline & b. & Aree & ri & alab'oom & x-e-kunax \\
\hline & & foc & les & enfants & ACC-IS3PL-soigner.PSF \\
\hline
\end{tabular}

4 Ce qui n'apparaît pas directement dans ces exemples, mais qui est essentiel pour valider l'utilisation de la notion de passif dans la description d'une langue (et notamment pour tracer une distinction entre passif et moyen), c'est que les formes verbales que comportent les phrases (b) des exemples ci-dessus assignent sans ambigüité à leur sujet un rôle sémantique exactement identique à celui que les formes verbales des phrases (a) assignent à leur objet : à la différence du moyen, le passif n'implique aucun remodelage des rôles sémantiques assignés par le verbe à ses arguments, mais simplement une réorganisation syntaxique des rôles que le verbe à la forme dite "active " assigne à son sujet et à son objet. En (3b), le fait qu'il y ait simplement manipulation syntaxique de rôles 
sémantiques qui ne subissent en eux-mêmes aucune modification est rendu évident par la présence du "complément d'agent ", qui récupère le rôle que la forme active du même verbe assigne à son sujet. En ( $(1 b)$ et $(2 b)$, en l'absence de " complément d'agent », ce qui prouve que ces formes verbales du tswana ou du peul signifient bien (en dehors de toute considération sur la situation de référence) que le référent du sujet subit l'action d'un agent, et ne signifient pas seulement que le sujet est le siège d'un processus, c'est que dans ces deux langues, les formes passives qui apparaissent dans ces phrases sont morphologiquement distinctes de formes moyennes dont l'utilisation aurait pour effet de présenter le référent du sujet comme comme le siège d'un processus plus ou moins spontané, ou en tout cas d'occulter l'éventuelle intervention d'une force extérieure dans le processus que subit le référent du sujet - ex. (4).

\begin{tabular}{|l|l|l|l|l|}
\hline (4) & tswana & & \multicolumn{2}{l|}{} \\
\hline & a. & \multicolumn{2}{|l|}{ Lokwalo } & \multicolumn{2}{l|}{ lo-tlaa-kwal-wa } \\
\hline & & 11 lettre & \multicolumn{2}{l|}{ ISCL11-FUT-écrire-PSF } \\
\hline & 'La lettre sera écrite' & lo-tlaa-kwal-ega \\
\hline & b. & Lokwalo & ISCL11-FUT-écrire-Mor \\
\hline & & 11lettre & \\
\hline & 'La lettre s'écrira' &
\end{tabular}

Il faut toutefois souligner ici que la possibilité d'introduction d'un complément d'agent ne doit pas être considérée comme décisive pour reconnaitre une construction comme passive. En effet, le peul est un cas typique de langue qui fait une distinction stricte, et marquée dans la totalité du paradigme verbal, entre formes passives et formes moyennes (seules les premières attribuant à leur sujet exactement le même rôle que celui que la forme transitive correspondante assigne à son objet), et pourtant c'est une langue dont les constructions passives ne peuvent jamais comporter un terme récupérant le rôle que la forme active du même verbe assigne à son sujet.

6 Cette définition du passif canonique étant posée, on observe que dans quantité de langues, les formes qui satisfont à cette définition dans une partie de leurs emplois ont aussi des emplois qui diffèrent du passif canonique, soit par la promotion d'un oblique au statut de sujet, soit par l'absence totale de sujet grammatical. Une façon de tenir compte de ceci consisterait à élargir la notion de passif de façon à pouvoir appliquer ce terme à toute formation morphologique régulièrement liée à un mécanisme syntaxique de destitution du sujet. Une telle décision risquerait toutefois d'être mal comprise et/ou difficilement acceptée, car, s'il va de soi de reconnaître des emplois non canoniques de passifs identifiables comme tels du fait de leur aptitude à entrer dans des constructions répondant à la définition du passif canonique, il n'est par contre pas usuel dans la description des langues d'étiqueter comme "passives» des formations morphologiques qui impliquent une destitution du sujet n'allant jamais de pair avec la promotion de l'objet. C'est pourquoi on s'en tiendra ici aux définitions suivantes : 
- une formation morphologique au niveau du verbe est identifiée comme voix passive si elle encode un mécanisme de destitution du sujet susceptible de s'accompagner de la promotion de l'objet des verbes transitifs ;

- on parlera de "passif canonique» lorsqu'une formation morphologique reconnue comme voix passive selon la définition précédente se trouve employée dans les constructions qui permettent de l'identifier comme telle- cf. ex. (1) à (3) ci-dessus ;

- on parlera de "passif oblique » lorsqu'une forme qui se prête par ailleurs à l'emploi passif canonique se trouve dans une construction où la destitution du sujet s'accompagne de la promotion d'un oblique - ex. (5b) ;

\begin{tabular}{|l|l|l|}
\hline (5) & \multicolumn{2}{|l|}{ anglais } \\
\hline & a. & Somebody has slept in this bed \\
\hline & b. & This bed has been slept in \\
\hline
\end{tabular}

7 - on parlera de " passif impersonnel » lorsqu'une forme qui se prête par ailleurs à l'emploi passif canonique ne s'accompagne d'aucune promotion; l'ex. (6c) illustre le passif impersonnel de verbes transitifs, et les ex. (7b-c) illustrent le passif impersonnel de verbes intransitifs.

\begin{tabular}{|c|c|c|c|c|c|c|c|c|c|c|}
\hline (6) & \multicolumn{9}{|c|}{ tswana } & \\
\hline & \multirow[t]{3}{*}{ a. } & Maburu & $a$ & \multicolumn{4}{|c|}{ rekile } & \multicolumn{2}{|c|}{ dikgomo } & \\
\hline & & 6Afrikaner & iscl6 & \multicolumn{4}{|c|}{ avoir+acheté } & \multicolumn{2}{|c|}{ 10bœuf } & \\
\hline & & \multicolumn{8}{|c|}{ 'Les Afrikaners ont acheté des bœufs' } & \\
\hline & \multirow[t]{3}{*}{ b. } & Dikgomo & \multicolumn{2}{|l|}{ di } & \multicolumn{3}{|c|}{ rekilwe } & (ke & Maburu) & \\
\hline & & 10bœuf & \multicolumn{2}{|c|}{ iscl10 } & \multicolumn{3}{|c|}{ avoir+acheté.PSF } & par & 6Afrikaner & \\
\hline & & \multicolumn{8}{|c|}{ 'Les bœufs ont été achetés (par des Afrikaners)' (passif canonique) } & \\
\hline & c. & Go & \multicolumn{4}{|c|}{ rekilwe } & \multicolumn{3}{|l|}{ dikgomo } & \\
\hline & & expl & \multicolumn{4}{|c|}{ avoir+acheté.PSF } & \multicolumn{3}{|l|}{ 10bœuf } & \\
\hline & & \multicolumn{8}{|c|}{ 'Des bœufs ont été achetés' (litt. Il a été acheté des bœufs) (passif impersonnel) } & \\
\hline \multirow[t]{4}{*}{ (7) } & \multicolumn{9}{|c|}{ allemand } & \\
\hline & a. & Die & \multicolumn{3}{|c|}{ Kinder } & \multicolumn{4}{|c|}{ schlafen } & \\
\hline & & les & \multicolumn{3}{|c|}{ enfants } & \multicolumn{4}{|c|}{ dorment } & \\
\hline & \multicolumn{9}{|c|}{ 'Les enfants dorment' } & \\
\hline
\end{tabular}




\begin{tabular}{|l|l|l|l|l|l|l|l|}
\hline & b. & Es & wird & von & den & Kindern & geschlafen \\
\hline & & il & est & par & les & enfants & dormi \\
\hline & & 'Les enfants dorment' (litt. Il est dormi par les enfants) & & & \\
\hline & c. & Es & wird & geschlafen & & & \\
\hline & & il & est & dormi & & \\
\hline & & \multicolumn{2}{|l|}{ 'On dort', 'Ça dort' (litt. Il est dormi) } & & \\
\hline
\end{tabular}

8 - on désignera comme "voix oblique » une formation morphologique qui signale une destitution de sujet s'accompagnant toujours de la promotion d'un oblique; on peut illustrer cette notion par les formes verbales du malgache traditionnellement désignées comme voix relative ou voix circonstancielle - ex. (8) ;

\begin{tabular}{|c|c|c|c|c|c|c|c|c|c|c|}
\hline (8) & malgache & & & & & & & & & \\
\hline & a. & Manasa & \multicolumn{4}{|l|}{ ny } & \multicolumn{2}{|c|}{ lamba } & \multicolumn{2}{|l|}{ Rasoa } \\
\hline & & laver & \multicolumn{4}{|l|}{ le } & \multicolumn{2}{|c|}{ linge } & \multicolumn{2}{|l|}{ Rasoa } \\
\hline & & \multicolumn{9}{|c|}{ 'Rasoa lave le linge' } \\
\hline & b. & Sasan & \multicolumn{3}{|l|}{-dRasoa } & \multicolumn{2}{|l|}{ ny } & \multicolumn{3}{|c|}{ lamba } \\
\hline & & Laver+par & \multicolumn{3}{|l|}{-Rasoa } & le & \multicolumn{4}{|c|}{ linge } \\
\hline & & \multicolumn{9}{|c|}{ 'Rasoa lave du linge avec le savon' } \\
\hline & c. & Manasa & \multicolumn{2}{|l|}{ lamba } & \multicolumn{2}{|l|}{ amin' } & \multicolumn{2}{|l|}{ ny } & savony & Rasoa \\
\hline & & laver & \multicolumn{2}{|l|}{ linge } & \multicolumn{2}{|l|}{ avec } & \multicolumn{2}{|l|}{ le } & savon & Rasoa \\
\hline & & \multicolumn{9}{|c|}{ 'Rasoa lave du linge avec du savon' } \\
\hline & d. & Anasan & -dRasoa & \multicolumn{3}{|l|}{ lamba } & \multicolumn{2}{|l|}{ ny } & \multicolumn{2}{|c|}{ savony } \\
\hline & & servir+à+laver+à & -Rasoa & \multicolumn{3}{|l|}{ linge } & le & \multicolumn{3}{|c|}{ savon } \\
\hline & & \multicolumn{9}{|c|}{ 'Le savon est utilisé par Rasoa à laver du linge' (voix oblique) } \\
\hline
\end{tabular}

9 - on désignera comme "voix impersonnelle » une formation morphologique signalant une destitution du sujet qui laisse toujours inchangé le reste de la construction du verbe. ${ }^{4}$ L'utilisation des mêmes formes verbales à la fois dans des constructions répondant à la définition du passif canonique et dans des constructions où la destitution du sujet ne s'accompagne d'aucun mécanisme de promotion semble notamment un phénomène 
extrêmement commun dans les langues du monde. Dans ce cas, les grammaires descriptives identifient généralement un passif impersonnel. Là où il y a par contre un problème, c'est lorsqu'on a dans une langue des formes verbales particulières dont l'emploi peut aller de pair, soit avec une destitution du sujet accompagnée de la promotion d'un oblique, soit avec une destitution du sujet non accompagnée de la promotion d'un quelconque autre terme, mais qu'on ne rencontre jamais dans des constructions répondant à la définition du passif canonique. On a en effet proposé ici les termes de «voix oblique » et "voix impersonnelle ", mais il n'y a dans la pratique des descriptions de langues aucun terme consacré qui permette immédiatement de reconnaître la présence de formes qui, même si elles ne se prêtent pas à des constructions du type passif canonique, partagent avec le passif la propriété d'encoder un mécanisme de destitution du sujet.

\section{La notion de moyen}

11 En généralisant la définition qui se dégage de l'usage du terme de moyen tel qu'il est traditionnellement utilisé dans la description de langues comme le grec ancien ou le peul, on peut convenir de désigner comme voix moyenne toute formation morphologique qui, lorsqu'elle s'applique à des verbes transitifs actifs prototypiques, permet d'obtenir une forme qui assigne à son sujet un rôle qui ne s'identifie totalement à aucun des deux rôles prototypiques d'agent et de patient. En d'autres termes, un élément essentiel des voix moyennes (notamment par contraste avec les voix passives) est que, lorsqu'un verbe possède à la fois une forme "active » et une forme moyenne, on ne peut pas décrire de façon satisfaisante la valence de la forme moyenne en termes de simples manipulations syntaxiques sur les rôles sémantiques que la forme active assigne à ses arguments : ce que la voix moyenne encode, c'est un remodelage du rôle sémantique assigné par le verbe au sujet, remodelage qui va dans le sensd'un brouillage de la distinction entre les rôles prototypiques d'agent et de patient. ${ }^{5}$

12 Les formations morphologiques reconnaissables comme voix moyennes selon cette définition se distinguent en outre des voix passives par les deux caractéristiques suivantes :

13 - Lorsque le verbe existe à la fois à la forme dite active et à la forme moyenne, il peut arriver qu'en ce qui concerne le nombre d'arguments nucléaires, la forme moyenne ait un effet de réduction qui rappelle le passif canonique - ex. (9); mais cela n'a rien de constant, et il est courant de trouver des verbes dont la forme active et la forme moyenne ne présentent aucune différence au niveau de la présence d'un objet et du rôle qui lui est assigné - ex. (10).

\begin{tabular}{|l|l|l|l|l|l|}
\hline (9) & peul & & & \multicolumn{2}{|}{} \\
\hline & a. & o & loot-ii & Eiyiko & \\
\hline & & iscl « o » & laver-Acc & enfant \\
\hline & & 'Il a lavé l'enfant' & \\
\hline & b. & o & loot-ake & \\
\hline
\end{tabular}




\begin{tabular}{|c|c|c|c|c|c|}
\hline & & iscl « o» & \multicolumn{3}{|l|}{ laver-AcC.MOY } \\
\hline & & \multicolumn{4}{|c|}{ 'Il s'est lavé' } \\
\hline (10) & peul & & & & \\
\hline & a. & 0 & res-ii & dum & \\
\hline & & iscl « o » & déposer-Acc & \multicolumn{2}{|c|}{ iocl »dum » } \\
\hline & & \multicolumn{4}{|c|}{ 'Il a déposé ça' } \\
\hline & b. & 0 & \multicolumn{2}{|l|}{ res-ake } & dum \\
\hline & & iscl « & déposer-АCс.MOY & \multicolumn{2}{|c|}{ iocl « dum » } \\
\hline & & \multicolumn{4}{|c|}{ 'Il s'est mis ça de côté' } \\
\hline
\end{tabular}

14 - Même lorsque cela n'est pas évident du point de vue morphologique, les formes passives peuvent de manière générale être considérées comme dérivées sur la base du fait qu'il est tout à fait exceptionnel de trouver des verbes qui ont la forme passive comme seule forme possible; il y a par contre dans toutes les langues où a été identifiée une voix moyenne une certaine proportion de verbes qui n'existent qu'à la forme moyenne (ou à la forme moyenne et à la forme passive, mais pas à la forme active, dans les systèmes qui distinguent ces trois voix, comme en peul). Par exemple, selon le comptage effectué par Arnott (repris dans Klaiman (1991)), 13,5\% des verbes peuls sont des intransitifs qui n'existent qu'à la voix moyenne, et $10 \%$ sont des transitifs qui n'existent qu'à la voix moyenne et à la voix passive.

Les formes moyennes ont généralement un éventail assez large de valeurs possibles selon le sens lexical des verbes, le seul élément qui fait l'unité de l'ensemble étant d'encoder un rôle du référent du sujet qui échappe à une stricte dichotomie agent / patient. D'un verbe à l'autre (et parfois pour un même verbe) le sujet peut présenter un degré très variable d'activité.

Le référent du sujet d'une forme moyenne peut être tout aussi actif qu'un agent prototypique, dont il se distingue dans ce cas par le fait qu'il est en même temps d'une manière ou d'une autre le lieu où se manifestent les effets de l'action, alors que dans le couple agent / patient prototypique, c'est le patient qui est le lieu de manifestation des effets de l'action (le patient subit typiquement un changement d'état, mais pas l'agent). Ce cas de figure englobe bien sûr comme cas particulier le réfléchi et le réciproque, qui font souvent partie des emplois que peuvent avoir les formes moyennes - ex. (9) cidessus, mais aussi le cas où le référent du sujet agit dans son propre intérêt - ex. (10) cidessus. Dans l'ex. (11), on n'a pas à proprement parler un sens de réfléchi, et le référent du sujet n'est pas celui qui fait l'action de tresser, mais il a indirectement un rôle actif (il a demandé à être tressé) et en même temps il est le lieu de la manifestation des effets de l'action. L'identification entre participant actif et lieu de manifestation des effets de l'action explique aussi la forme moyenne de verbes qui en peul n'existent pas à la voix active - ex. (12). 


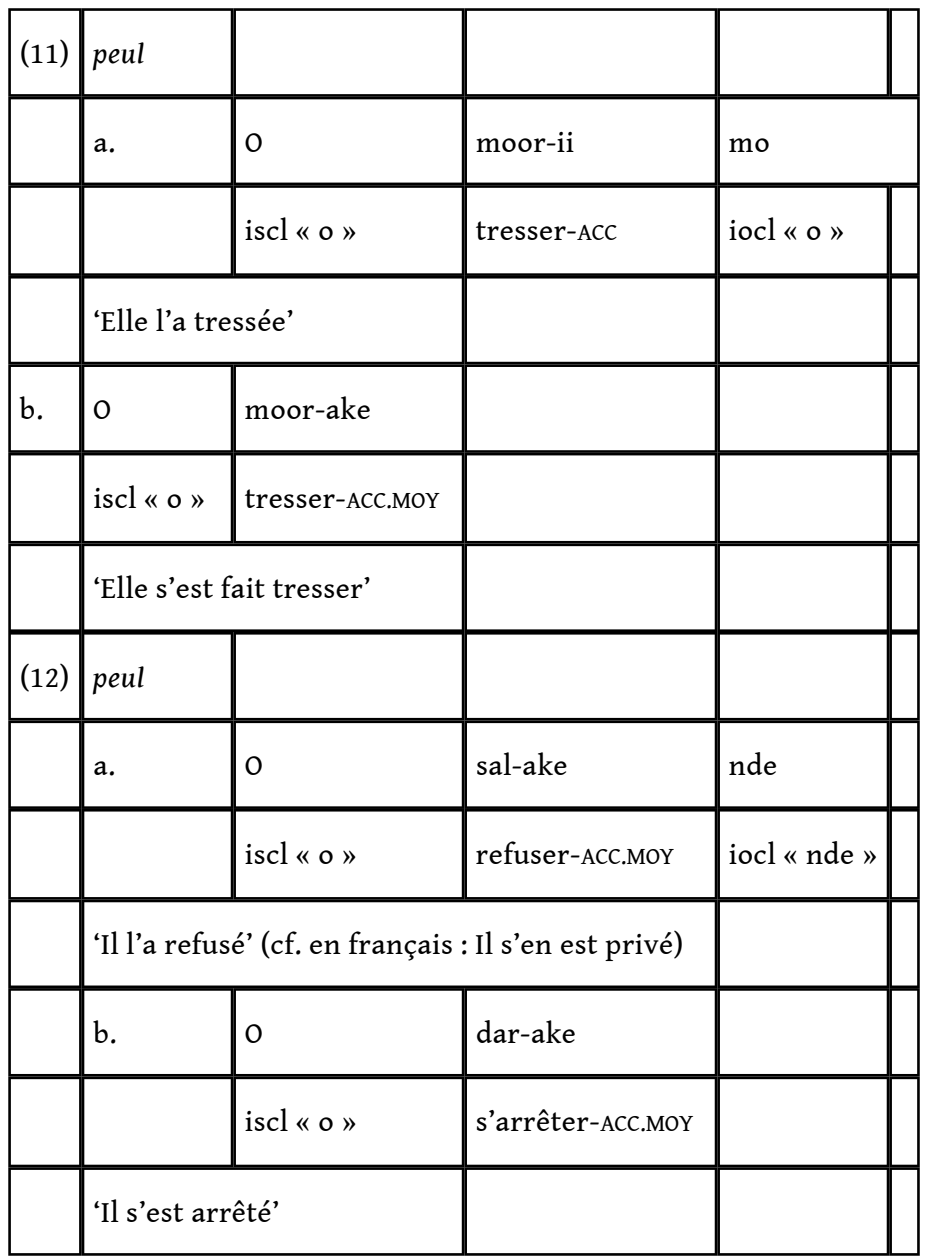

17 Mais le référent du sujet d'une forme moyenne peut aussi être tout aussi passif qu'un patient prototypique, dont il va alors se distinguer par le caractère plus ou moins spontané (ou présenté comme tel) du processus qui l'affecte, alors que le patient prototypique subit l'action d'une force extérieure. On peut retenir le terme de "décausatif» pour cet emploi des formes moyennes, parfois désigné aussi comme «neutre »- ex. (13).

\begin{tabular}{|l|l|l|l|l|}
\hline (13) & peul & & & \\
\hline & a. & O & Eill-ii & dum \\
\hline \hline & & iscl « o » & écraser-Acc & iocl « dum » \\
\hline \hline & 'Il a écrasé ça' & & \\
\hline & b. & 'Dum & Eill-ake & \\
\hline & & iscl « dum » & écraser-ACc.MOY & \\
\hline \hline & 'Ça s'est écrasé' & & & \\
\hline
\end{tabular}


L'emploi décausatif des formes moyennes a une affinité évidente avec les notions aspectomodales de potentialité et d'habitude, et un emploi typique des formes moyennes est de présenter l'aptitude ou la prédisposition à subir un processus ou une action comme une propriété caractéristique du référent du sujet - ex. (14).

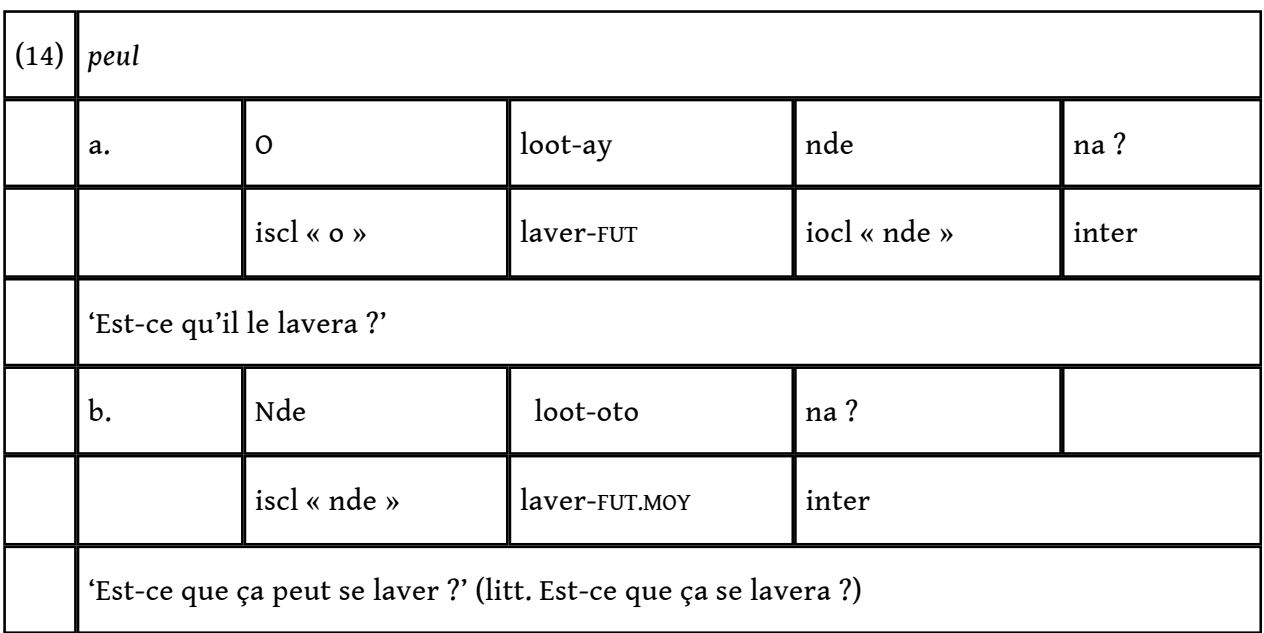

Lorsqu'on utilise des grammaires descriptives dans une perspective typologique, il faut être attentif au fait que, si les descripteurs de langues ont tendance à abuser du terme de passif (nous reviendrons là-dessus à la section III), par contre le terme de voix moyenne n'est utilisé traditionnellement que dans la description d'une faible proportion de langues parmi celles qui ont une formation morphologique répondant à la définition de la voix moyenne. Les formes moyennes sont la plupart du temps,

- ou bien désignées comme " passives",

- ou bien désignées comme " réfléchies ${ }^{6}$

- ou bien encore désignées de termes qui ne font aucune référence à leur statut dans l'organisation de la valence verbale (formes «pronominales» des langues romanes ou slaves, formes «faibles» du tamoul, «ikes igék» (verbes en -ik) du hongrois, etc.); en particulier, les «formes pronominales» du verbe français ont tout d'une voix moyenne typique, et les «formes pronominales de sens passif » de la grammaire scolaire du français sont plutôt des formes moyennes en emploi décausatif (ce qui constitue un emploi sémantiquement proche du passif mais tout de même distinct).

\section{Moyen, passif et médio-passif}

Il convient maintenant d'envisager une situation apparemment très commune dans les langues du monde, dans laquelle une même formation morphologique peut se rencontrer à la fois dans des constructions impliquant une opération sur la valence verbale répondant à la notion de passif (réarrangement syntaxique de rôles sémantiques qui en eux-mêmes ne subissent aucune modification) et dans des constructions impliquant une opération sur la valence verbale répondant à la notion de moyen (remodelage du rôle sémantique du sujet). Pour traiter sans contradiction de telles situations, il importe d'accepter l'idée que le fait de pouvoir rencontrer une forme verbale dans une construction identifiable comme passive ne signifie pas nécessairement que cette forme puisse être identifiée dans l'absolu comme passive. 
21 Par exemple, dans la phrase latine (15b) et dans la phrase peule (16b), on peut également parler de constructions passives canoniques, dans la mesure où la promotion de l'argument traité comme objet en (15a) ou (16a) s'accompagne d'une modification morphologique du verbe. Mais il y a un problème à désigner la forme verbale qui apparaît en (15b) comme passive, car en latin - ex. (15c-e), les mêmes formes du verbe apparaissent dans des constructions où il n'est pas possible d'analyser le sujet en termes de promotion de l'objet d'une construction active. Par contre en peul, en dépit de l'impossibilité d'introduire dans la construction un complément d'agent, il n'y a aucun problème à désigner comme passive la forme verbale qui apparait en (16b) ; en effet, comme l'illustre la comparaison avec (16c), le peul fait une distinction stricte et qui traverse la totalité du paradigme verbal entre des formes passives (dont le sujet est toujours sémantiquement identifiable à l'objet d'une construction active correspondante) et des formes moyennes (aptes à exprimer des valeurs qui ne relèvent pas de la notion de passif, mais qui en latin pouvaient parfois s'exprimer par des formes identiques à celles rencontrées dans les constructions passives).

\begin{tabular}{|c|c|c|c|c|c|c|}
\hline (15) & latin & & & & & \\
\hline & a. & Magister & \multicolumn{2}{|l|}{ pueros } & \multicolumn{2}{|l|}{ laudat } \\
\hline & & maître.sG & \multicolumn{2}{|l|}{ garçon.oBJ.PL } & \multicolumn{2}{|l|}{ féliciter.3SG } \\
\hline & & \multicolumn{5}{|c|}{ 'Le maître félicite les garçons' } \\
\hline & b. & Pueri & $\mathrm{a}$ & magistro & & laudantur \\
\hline & & garçon.PL & par & maître.ABL.Sc & & être+félicité.3PL \\
\hline & & \multicolumn{5}{|c|}{ 'Les garçons sont félicités par le maître' } \\
\hline & c. & Pueri & \multicolumn{4}{|c|}{ exercebantur } \\
\hline & & garçon.PL & \multicolumn{4}{|c|}{ s'exercer.IMPF.3PL } \\
\hline & & \multicolumn{5}{|c|}{ 'Les garçons s'exerçaient' } \\
\hline & d. & Copulantur & \multicolumn{4}{|l|}{ dexteras } \\
\hline & & se+serrer.3PL & \multicolumn{4}{|c|}{ main+droite.oBJ.PL } \\
\hline & & \multicolumn{5}{|c|}{ 'Ils se serrent la main' } \\
\hline & e. & Lætantur & & & & \\
\hline & & se+réjouir.3PL & & & & \\
\hline & & \multicolumn{5}{|c|}{ 'Ils se réjouissent' } \\
\hline (16) & peul & & & & & \\
\hline
\end{tabular}




\begin{tabular}{|l|l|l|l|l|}
\hline & a. & O & mооБt-ii & Бе \\
\hline & & iscl »o » & rassembler-ACC & iocl »be » \\
\hline & & 'Il les a rassemblés' \\
\hline & b. & 'Be & mooБt-aama \\
\hline \hline & & iscl « be » & rassembler-ACC.PSF \\
\hline & & 'Ils ont été rassemblés' (passif) \\
\hline & c. & 'Be & mooБt-iima \\
\hline & & ISCL «BE» & rassembler-ACC.MOY \\
\hline & & 'Ils se sont rassemblés' (moyen) \\
\hline
\end{tabular}

Du point de vue terminologique, il serait utile dans la perspective d'une description synchronique de retenir un terme comme par exemple médio-passif pour étiqueter les formes verbales (très communes dans les langues du monde) aptes à figurer également dans des constructions relevant de la notion de moyen et dans des constructions relevant de la notion de passif. Selon cette terminologie, les formes «passives » du latin seraient plutôt des formes médio-passives, et un examen critique des grammaires descriptives révèle qu'on peut en dire autant d'un nombre considérable de langues dans lesquelles on identifie traditionnellement comme passives des formes qui sont en réalité médiopassives (c'est notamment le cas du « get-passive » de l'anglais).

\section{Du moyen au passif}

$23 \mathrm{Au}$ moins deux types distincts d'évolutions historiques sont très largement attestés comme origine possible de constructions passives : passifs issus de moyen et passifs issus de statif-résultatif.?

24 Le fait qu'on rencontre très couramment dans les langues du monde des formes verbales reconnaissables comme médio-passives selon la définition proposée à la section III suggère qu'il doit y avoir des possibilités de réinterpréter comme passives des constructions moyennes, ou peut-être l'inverse, et que cette possibilité se concrétise fréquemment dans l'évolution des langues.

En ce qui concerne le sens du changement, les données dont on dispose laissent penser que l'évolution se fait du moyen vers le passif plutôt qu'en sens inverse : des formes dont le domaine d'emploi initial se situe strictement dans le domaine de la voix moyenne acquièrent d'abord des emplois de type passif, et peuvent dans une étape ultérieure tendre à se spécialiser dans ce type d'emploi et à perdre les emplois de type moyen qu'elles assumaient initialement.

Nous avons vu ci-dessus qu'un emploi typique des formes moyennes est l'emploi décausatif, dans lequel le référent du sujet est présenté comme le lieu de manifestation d'un événement plus ou moins spontané, c'est-à-dire n'impliquant aucune cause 
extérieure clairement identifiable. La proximité sémantique entre le rôle assigné au sujet dans cet emploi des formes moyennes et celui assigné au sujet de la forme passive des verbes d'action transitifs rend la distinction entre moyen et passif particulièrement perméable. La perméabilité est d'autant plus grande que souvent, l'utilisation de la forme moyenne implique de passer en quelque sorte sous silence la participation d'un agent sans l'intervention duquel on ne saurait concevoir le procès. Par exemple, en (17a), les formes moyennes dénotent bien des processus dans lesquels on n'isole pas de façon nette l'action d'une force extérieure bien identifiable; par contre, en (17b), il est clair qu'on peut difficilement parler de référence à des processus dont la cause extérieure est inexistante ou difficilement identifiable, et qu'il y a plutôt occultation de l'agent en liaison avec une signification du type « aptitude du référent du sujet à subir l'action ».

\begin{tabular}{|l|l|l|}
\hline (17) & \multicolumn{2}{|l|}{ français } \\
\hline \hline & a. & Ce tissu se froisse / se salit / s'use très vite \\
\hline & b. & Ce tissu se lave / se coud / se repasse facilement \\
\hline
\end{tabular}

A partir d'emplois du type illustré en (17b), on conçoit aisément que, selon un mécanisme décrit par les théoriciens de la grammaticalisation sous le nom de « sémantisation d'une inférence pragmatique", des formes moyennes puissent acquérir des emplois proprement passifs, c'est-à-dire des emplois dans lesquels le sujet se voit assigner un rôle exactement identique à celui que la forme active du même verbe assigne à son objet. Cette évolution peut s'observer avec les formes « pronominales » du russe, de l'espagnol ou de l'italien. En effet, à la différence du français (où on peut soutenir que les formes "pronominales » dites «de sens passif» n'ont jamais un sens exactement identique à celui des formes passives du même verbe), il y a en russe, en espagnol ou en italien des cas où les formes pronominales assument indiscutablement des emplois véritablement de type passif, c'est-à-dire qui se laissent décrire comme le résultat de simples manipulations syntaxiques sur les rôles sémantiques assignés par la forme active correspondante - ex. (18). C'est ce que montre clairement, en (18a) et (18b), la possibilité d'un complément d'agent. Dans le cas de l'espagnol, on ne peut pas utiliser le même argument (car les formes pronominales de l'espagnol, même dans leurs emplois proprement passifs, restent incompatibles avec un complément d'agent), mais on peut remarquer la différence entre la phrase espagnole (18c) et son correspondant littéral en français : en français, la seule interprétation possible de la forme pronominale serait dans ce cas l'interprétation réfléchie ou réciproque ; en espagnol par contre, l'interprétation réfléchie ou réciproque reste certes possible, mais l'interprétation passive est celle qui vient la première à l'esprit.

\begin{tabular}{|l|l|l|l|l|l|}
\hline$(18)$ & & \multicolumn{4}{l|}{ russe (a) / italien (b) / espagnol (c) } \\
\hline & a. & Eto & javlenie & $\begin{array}{l}\text { issledujet- } \\
\text { sja }\end{array}$ & ucčenymi \\
\hline & & ce & phénomène & étudie-MoY & scientifiques.INSTR \\
\hline
\end{tabular}




\begin{tabular}{|c|c|c|c|c|c|c|c|}
\hline & \multicolumn{7}{|c|}{$\begin{array}{l}\text { 'Ce phénomène est étudié (litt. } \\
\text { s'étudie) par les scientifiques' }\end{array}$} \\
\hline b. & Questo & giornale & si & legge & $\mathrm{da}$ & moltissima & gente \\
\hline & ce & journal & moy & lit & par & $\begin{array}{l}\text { très } \\
+ \text { nombreux }\end{array}$ & gens \\
\hline & \multicolumn{7}{|c|}{ 'Ce journal est lu (litt. se lit) par de très nombreuses personnes' } \\
\hline c. & $\mathrm{Se}$ & invitaron & muchos & \multicolumn{4}{|c|}{ amigos } \\
\hline & moy & invitèrent & nombreux & \multicolumn{4}{|l|}{ amis } \\
\hline & 'Beau & été in & vitèrent)' & & & & \\
\hline
\end{tabular}

C'est probablement ce scénario, que nous voyons se dérouler dans les langues romanes modernes, qui s'était déjà produit dans l'histoire du latin, puisque les formes du latin couramment désignées comme passives résultent d'une évolution de la voix moyenne de l'indo-européen. Simplement, les formes pronominales des langues romanes modernes attestent la phase initiale d'une telle évolution, alors qu'au stade du latin, l'évolution était apparemment suffisamment avancée pour les grammairiens considèrent comme synchroniquement plus ou moins marginaux (à tort ou à raison, on ne cherchera pas à trancher ici) les emplois de type moyen encore conservés par les formes issues du moyen indo-européen.

\section{L'origine des formes passives et la question de l'affinité entre passif et « stativisation »}

29 A côté de passifs issus comme cela vient d'être évoqué de la réanalyse de l'emploi décausatif de formes moyennes, on trouve aussi très couramment des passifs qui proviennent de toute évidence de la réanalyse de constructions statives-résultatives, mettant typiquement en jeu une copule de sens statif et une forme dérivée de verbe à valeur résultative (comme être + participe passé en français).

Les processus de réanalyse de la valeur d'une construction sont des processus progressifs: la valeur d'une construction ne change pas brutalement du jour au lendemain, et il y a toujours une période plus ou moins longue où l'interprétation originelle et celle qui est en train de se développer se trouvent en concurrence. On peut donc s'attendre à ce que, au moins pendant un certain temps, les formes composées du type copule stative + forme résultative du verbe qui tendent à acquérir le statut de formes passives conservent au moins avec certains verbes la possibilité de s'utiliser avec leur valeur stative-résultative initiale.

31 C'est une telle situation qui s'observe actuellement en français, ${ }^{8}$ et dans pas mal d'autres langues (dont l'anglais). C'est ce qui explique que, dans la description d'un assez grand nombre de langues, on utilise couramment le terme de passif pour se référer à des constructions statives-résultatives, en précisant seulement qu'il peut y avoir à distinguer entre " passif d'état » et " passif d'action », ou entre « faux passif » et « vrai passif ». Cette 
pratique terminologique s'observe même dans le cas de langues où (comme par exemple en allemand) les constructions statives résultatives sont formellement distinctes du véritable passif.

La thèse défendue ici est que le statif-résultatif n'est pas une variété de passif, mais plutôt une source possible du passif, et que l'ambigüité observée dans de nombreuses langues entre passif et statif-résultatif ne tient pas à une propriété intrinsèque des passifs, mais au fait que les constructions passives qui ont pour source une construction stativerésultative peuvent conserver au moins un certain temps une possibilité d'interprétation de type statif-résultatif.

Cette position se situe à l'opposé de la thèse de Givón (cf. Givón (1990), chapitre 14) selon laquelle les formes verbales passives tendraient universellement à présenter des particularités d'ordre aspectuel qu'il résume du terme de "stativisation ». Mais on n'en finirait plus d'énumérer les langues possédant des formes passives qu'aucune particularité aspectuelle de ce type ne distingue des formes actives correspondantes, et ceci appelle deux commentaires.

Tout d'abord, on ne doit pas s'attendre à ce que des passifs issus de moyen se distinguent par de telles particularités aspectuelles, car la seule dimension aspecto-modale qui semble avoir régulièrement une affinité avec le moyen est la potentialité. On peut évoquer à ce sujet le cas du russe, qui a un passif issu de résultatif pour les verbes perfectifs et un passif issu de moyen pour les imperfectifs.

Ensuite, même en ce qui concerne les passifs issus de résultatif, l'examen attentif des faits d'une langue comme l'espagnol suggèrent que l'ambigüité entre passif et statif-résultatif peut disparaitre du fait d'évolutions ultérieures, ce qui ne devrait pas se produire s'il y avait effectivement une relation naturelle entre passif et «stativisation ». En effet, en espagnol, le passif ser + participe passé, dont l'origine résultative est évidente, n'a plus d'emploi de type résultatif, et la signification stative-résultative s'exprime sans ambigüité en espagnol au moyen de estar + participe passé - ex. (19).

\begin{tabular}{|l|l|l|l|}
\hline$(19)$ & & \multicolumn{2}{|l|}{ français / espagnol } \\
\hline & a. & $\begin{array}{l}\text { La puerta está abierta } \\
\text { 'La porte est ouverte' }\end{array}$ & (résultatif) \\
\hline & $\begin{array}{l}\text { b. } \\
\text { La puerta es abierta por el portero } \\
\text { 'La porte est ouverte par le concierge' }\end{array}$ & (passif) \\
\hline
\end{tabular}

L'explication est que ser est une copule ancienne (qui avait déjà le statut de copule en latin), qui se trouve en concurrence avec une copule historiquement récente (estar), issue d'un verbe de posture du latin. Cette nouvelle copule a acquis progessivement une partie des emplois assumés initialement par ser, et elle a notamment servi à recréer une construction résultative estar + participe passé synchroniquement distincte de la construction ser + participe passé. De ce fait, la construction ser + participe passé exprime sans ambigüité une signification passive qui ne manifeste pas d'interaction particulière avec les significations aspectuelles. 


\section{BIBLIOGRAPHIE}

CREISSELS, Denis (2000) «L'emploi résultatif de être + participe passé en français », in Cahiers Chronos $6: 133-142$

GENIUŠIENE, Emma (1987) The typology of reflexives, Mouton de Gruyter, Berlin.

GIVÓN, Talmy (1990) Syntax, a functional-typological introduction, volume II, John Benjamins, Amsterdam.

KEMMER, Suzanne (1993) The middle voice, John Benjamins, Amsterdam.

KLAIMAN, Miriam (1991) Grammatical voice, Cambridge University Press, Cambridge.

\section{NOTES}

1. Dans ce qui suit, on utilisera par abréviation l'expression « destitution du sujet » pour signifier qu'en liaison avec une modification morphologique au niveau du verbe, l'argument qui en l'absence de cette modification morphologique serait sélectionné comme sujet syntaxique n'a plus ce statut, soit qu'il disparaisse complètement de la construction, soit qu'il soit " récupéré " avec un statut syntaxique autre que sujet.

2. Dans ce qui suit, on utilisera par abréviation l'expression "promotion de l'objet » pour signifier qu'en liaison avec une modification morphologique au niveau du verbe, le sujet représente l'argument qui en l'absence de cette modification morphologique serait sélectionné comme objet syntaxique.

3. Les abréviations suivantes sont utilisées dans les gloses des exemples: $A B L=$ ablatif, $A C C=$ accompli, $\mathrm{CL}=$ classe, $\mathrm{EXPL}=$ explétif, $\mathrm{FUT}=$ futur, $\mathrm{IMPF}=$ imparfait, $\mathrm{INSTR}=$ instrumental, $\mathrm{IO}=$ indice d'objet, $\mathrm{IS}=$ indice de sujet, $\mathrm{MOY}=$ moyen, $\mathrm{OBJ}=$ objet, $\mathrm{PL}=$ pluriel $\mathrm{PSF}=$ passif, $\mathrm{SG}=$ singulier $;$ un chiffre, s'il figure à l'initiale d'une forme nominale ou s'il succède immédiatement à cL, indique la numérotation d'une classe nominale selon la numérotation traditionnelle des classes nominales bantoues, autrement il indique une personne; les classes nominales du peul sont étiquetées d'après l'indice de sujet qui caractérise chaque classe $: C L$ « $O$ », $C L$ « $B E »$, etc.

4. Il y a de bons arguments pour considérer que le on du français, lorsqu'il n'est pas synonyme de nous, devrait être analysé comme marqueur d'une voix impersonnelle. En effet, on est traditionnellement analysé comme pronom indéfini en fonction de sujet, mais il n'a pas les propriétés discursives caractéristiques d'un pronom indéfini qui introduit dans le discours un référent susceptible d'être ultérieurement repris. L'impossibilité d'avoir par exemple *On ${ }_{i}$ a laissé son $_{i}$ adresse pour que tu lui $i_{i}$ écrives de la même façon qu'on a Quelqu'un $n_{i}$ a laissé son ${ }_{i}$ adresse pour que tu lui $i_{i}$ écrives suggère de considérer le on indéfini, non pas comme un indice pronominal sujet, mais plutôt comme un morphème encodant une opération sur la valence verbale.

5. Sur la problématique générale de la voix moyenne, cf. notamment Kemmer (1993) et Klaiman (1991) ; cf. aussi Geniušienè (1987), qui sous l'intitulé A typology of reflexives présente à peu de choses près une typologie des voix moyennes; en effet, elle englobe dans son étude toutes les formations morphologiques aptes à exprimer le réfléchi, ou issues d'anciens réfléchis ; or il est peu courant de rencontrer des voix moyennes qui ne sont, ni synchroniquement aptes à exprimer le réfléchi, ni issues d'anciens réfléchis. 
6. cf. notamment, note 5 , la remarque à propos de Geniušienè (1987).

7. On peut peut-être rajouter comme troisième source possible les constructions à sujet indéfini, mais les constructions à sujet indéfini semblent être à l'origine de voix impersonnelles plutôt que de véritables voix passives : cette possibilité est en effet évoquée par Givón (cf. Givón (1990), chapitre 14), mais cet auteur opère avec une définition très large de la notion de voix en général et de passif en particulier, et les exemples bantous qu'il fournit comme illustration de passifs issus de constructions à sujet indéfini, si on les analyse selon les définitions retenues ici, relèvent plutôt de la notion de voix impersonnelle. Par ailleurs, Givón parle aussi de passifs issus de réfléchis, mais il semble évident que la relation entre réfléchi et passif n'est pas directe : c'est seulement dans la mesure où des formes réfléchies étendent d'abord leurs emplois pour prendre le statut de voix moyenne qu'elles peuvent dans une étape ultérieure acquérir des emplois de type passif.

8. cf. Creissels (2000).

\section{AUTEUR}

\section{DENIS CREISSELS}

Denis.Creissels@univ-lyon2.fr 